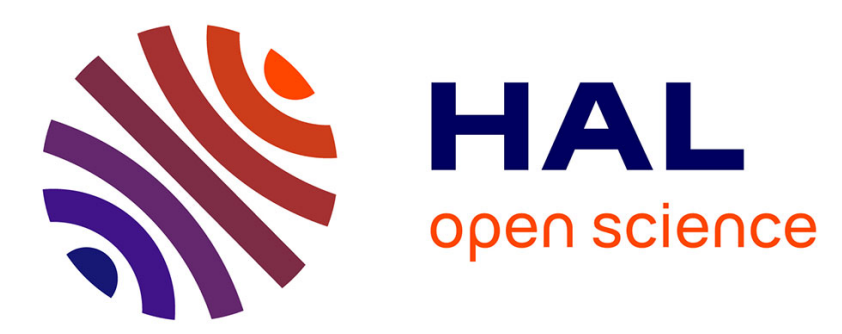

\title{
Fast MIP Handover Amelioration in Wireless Networks by Cross-Layer Solution
}

\author{
Anne Wei, Gouzhi Wei, Gerard Dupeyrat, Benoit Geller
}

\section{To cite this version:}

Anne Wei, Gouzhi Wei, Gerard Dupeyrat, Benoit Geller. Fast MIP Handover Amelioration in Wireless Networks by Cross-Layer Solution. Next Generation Mobile Applications, Services and Technologies, 2008. NGMAST '08. The Second International Conference on, Sep 2008, Cardiff, United Kingdom. pp.131-135, 10.1109/NGMAST.2008.57 . hal-01229106

\section{HAL Id: hal-01229106 \\ https://hal-ensta-paris.archives-ouvertes.fr/hal-01229106}

Submitted on 16 Nov 2015

HAL is a multi-disciplinary open access archive for the deposit and dissemination of scientific research documents, whether they are published or not. The documents may come from teaching and research institutions in France or abroad, or from public or private research centers.
L'archive ouverte pluridisciplinaire $\mathbf{H A L}$, est destinée au dépôt et à la diffusion de documents scientifiques de niveau recherche, publiés ou non, émanant des établissements d'enseignement et de recherche français ou étrangers, des laboratoires publics ou privés. 


\title{
Fast MIP Handover Amelioration in Wireless Networks by Cross-layer Solution
}

\author{
Anne Wei, ${ }^{\dagger}$ GouZhi Wei, ${ }^{*}$ Gerard Dupeyrat* and Benoit Geller ${ }^{\ddagger}$ \\ *Université Paris XII, 61 avenue du Général de Gaulle, 94030 Créteil, France \\ $\dagger$ Conservatoire National des Arts et Métiers, 292, rue Saint-Martin, 75003,Paris,France \\ ${ }^{\ddagger}$ SATIE - ENS Cachan, 61 avenue du Président Wilson, 94235 Cachan Cedex,France \\ Email: anne.wei@cnam.fr
}

\begin{abstract}
Mobile IP allows a mobile node to maintain a continuous connectivity to the Internet when moving from one access point to another. However, due to the link switching operations packets designated to mobile nodes can be delayed or lost during the handover period. This paper presents a twolayer solution to improve the handover performance both at the Link Layer and the Network Layer in the context of Mobile IP over wireless networks. At the Network Layer, we use a new function named Extended Handover Control Function $(\mathrm{EHCF})$ which allow us to delete the $D A D$ operation. At the Link Layer, a neighbor graphical prediction approach $(N G P)$ reduces the probe latency. Moreover, the $E H C F$ can buffer the packets during the handover process in order to decrease the packet loss. With an analytical model and some OPNET simulations, we show in this paper that our solution allows to provide low latency, low packet loss to the standard handover of Mobile IPv6.
\end{abstract}

Index Terms - Cross layer, Fast Handover and Mobile IPv6

\section{INTRODUCTION}

$\mathbf{T}$ HE need to keep an "everywhere and at any time" connection with Internet has been more and more demanded in recent years with the success of IEEE 802.11 and of IEEE 802.16 wireless networks standards. A growing number of $802.16 / 802.11$ based wireless networks has been deployed as access networks to the Internet. With those access networks, the mobility support has thus become possible. However, the continuous Internet connectivity and the correct routing of packets were not guaranteed when users change their access points to Internet with classical protocols. To resolve these problems, the Mobile IPv4 (MIPv4) and Mobile IPv6 (MIPv6)protocols [1], [2] were respectively published by the Internet Engineering Task Force (IETF). MIPv6 works well when Nomad users connects spontaneously at Internet without a continuous move. If an user continuously change its access points, the high handover latency and the high packet loss provide some troubles to support the continuous connection with MIP.

As described in $M I P v 6$, the handover latency consists of the link latency and the network latency caused both at the Link Layer and Network Layer. According to some studies [7], [19], it is found that the handover latency normally takes hundreds of milliseconds due to the probe at the Link Layer

This work was supported in part by the international project PRA-SIP under Grant SIP04-03. and more one second due to the $D A D$ (Detection Address Duplication) operation at the Network Layer.

Since 2003 [20], the main proposals by the IETF and the IEEE are the Hierarchical Mobile IPv6 (HMIPv6) and the Fast Handover for MIPv6 (FHMIPv6). HMIPv6 introduces a Mobility Anchor Point $(M A P)$ who acts somehow like a local Home Agent $(H A)$ for the visiting Mobile Node $(M N)$. The concept of MAP can limit the amount of signaling required outside the MAP's domain at the Link Layer [5], [7]. While FHMIPv6 used location-based fast handover with the InterAccess Point Protocol (IAPP) at the Link Layer [8], [14], [22]. The network uses a Link Layer trigger to launch either PreRegistration or Post-Registration handover operations. Besides of these main proposals, there has been also some approaches for providing the lossless handover and minimizing the handover delay [9]-[12]. In [9], a Pre-Handover Signaling (PHS) protocol is proposed in order to support the triggering of a predictive handover and to allow the network to achieve accurate handover decisions by considering different constraints such as Quality-of-Service $(Q o S)$, user profile and mobile node service requirements. In [10], a Hierarchical Network-layer Mobility Management (HNMM) framework is described in which an integrated IP-layer handover solution provides an optimized network connectivity. Also, a Competition based Soft Handover Management (CSHM) protocol [11] and a Multi-path Transmission Algorithm (MTA) [12] have been presented to decrease packet loss during a handover.

The goal of this paper is to optimize the Mobile IPv6 handover procedure both at the Link Layer and the Network Layer. At the Network Layer, we use a new function named Extended Handover Control Function (EHCF) which allow us to delete the $D A D$ operation. At the Link Layer, a neighbor graphical prediction approach reduces the probe latency. Moreover, the $E H C F$ can buffer the packets during the handover process in order to decrease the packet loss.

The remainder of the paper is thus organized as follows: Section II presents both the Neighbor Graphical Prediction $(N G P)$ and the Extended Handover Control Function $(E H C F)$ approaches with the associated operations. Then we describe the cross-layer solution with the NGP and EHCF approaches. Section III deals with the handover performance in terms of handover latency and packet loss. Regarding the standard handover of MIPv6, our numerical results show that our cross-layer solution NGP-EHCF reduces significantly both the 
latency and the packet loss. Finally, some conclusions are drawn in Section IV.

\section{CRoss-LAYER Solution With THE NGP AND THE EHCF}

\section{A. Handover latency overview}

Generally speaking, a handover consists of a Link Layer handover and a Network Layer handover. The Link Layer handover includes a Discovery phase (scanning the channels to discover an available Access Point), an Authentication phase, and a Re-association phase, whereas the Network Layer handover concerns a Router Discovery phase, a Detection Address Duplication $(D A D)$ phase, a Binding Update phase and a Binding Acknowledgement phase respectively. As displayed on Figure 1, the standard MIPv6 handover latency has been estimated to a maximum value of $1620 \mathrm{~ms}$ [7], [19]. This high latency is not acceptable for real time applications such as video and audio. If we analyze each phase during the handover process, we can note that the probe provides a $240-360 \mathrm{~ms}$ latency at the Link Layer and the $D A D$ latency costs almost $1000 \mathrm{~ms}$ at the Network Layer.

\begin{tabular}{|c|c|c|}
\hline & Link Layer handover \\
\hline Probe & Authentication & Re-association \\
\hline $240-360 \mathrm{~ms}$ & $10 \mathrm{~ms}$ & $10 \mathrm{~ms}$ \\
\hline
\end{tabular}

\begin{tabular}{|l|c|c|c|}
\hline \multicolumn{4}{|c}{ Network Layer handover } \\
\hline Router Discovery & DAD & Binding Update & Binding Acknowledgement \\
\hline $100 \mathrm{~ms}$ & $1 \mathrm{~s}$ & $70 \mathrm{~ms}$ & $70 \mathrm{~ms}$
\end{tabular}

Fig. 1. Standard MIPv6 Latency both at the Link Layer and at the Network Layer

During a handover, the Probe phase allows a MN to scan all nearby APs (Access Point) and then to choice it self the best channel for its new connection. The probe latency depends on the number of channels (in the IEEE 802.11 case; the number of channels scanned is about 11). The idea to reduce the number of channels scanned or to predict a good channel is studied in [20]-[22] indicated in the Section I.

\section{B. Neighbor Graphical Prediction-NGP at the Link Layer}

To reduce the number of channels scanned, two approaches (active and passive) have been used. With the active approach, a MN decides which is the channel for the next connection. In this case, the MN chooses the first scanned channel with enough energy without scanning any further channels. On the contrary, with the passive approach, an external server indicates the channels that the MN can scan [21].

We present a Neighbor Graphical Prediction approach (NGP) being based on a hierarchical network architecture which is illustrated on Figure 2. Directly linked with its ARs/APs/MNs, each $E H C F$ router can collect all transit data coming from each entity.

Assuming that an EHCF router charges $30 \mathrm{APs}$ as shown on Figure 3, the NGP approach allows an EHCF router to

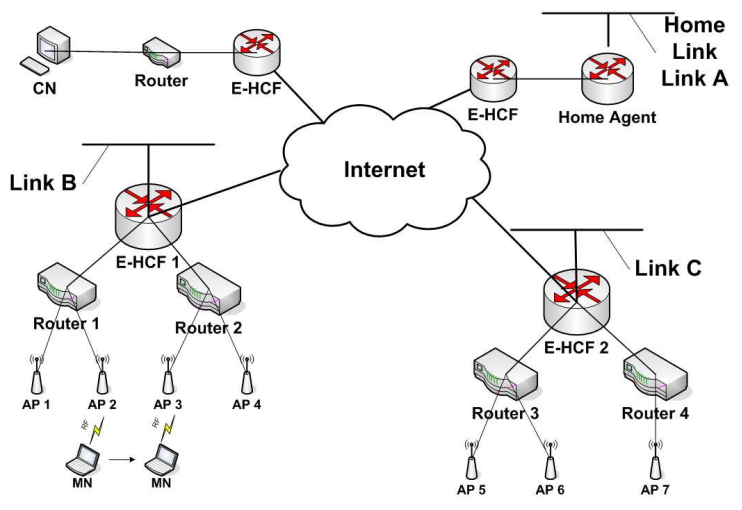

Fig. 2. Architecture of Fast Handover in IEEE 802.11 Wireless Networks(Router is an Access Router; EHCF is an EHCF router)

predict the next access point for the moving $\mathrm{MN}$ if the next access point is found (see Figue4).

The NGP method works as the following: when a MN moves from an $A P_{i}$ (for example, $A P_{i}=21$ ) to another $A P_{j}$ (for example, $A P_{j}=25$ ), it indicates its $E H C F$ router that triggers a handover. According to the graphical map, the $E H C F$ router then launches the NGP-A algorithm to decide which is the next access point for the moving MN (the procedure of the EHCF router is illustrated on Figure 5).

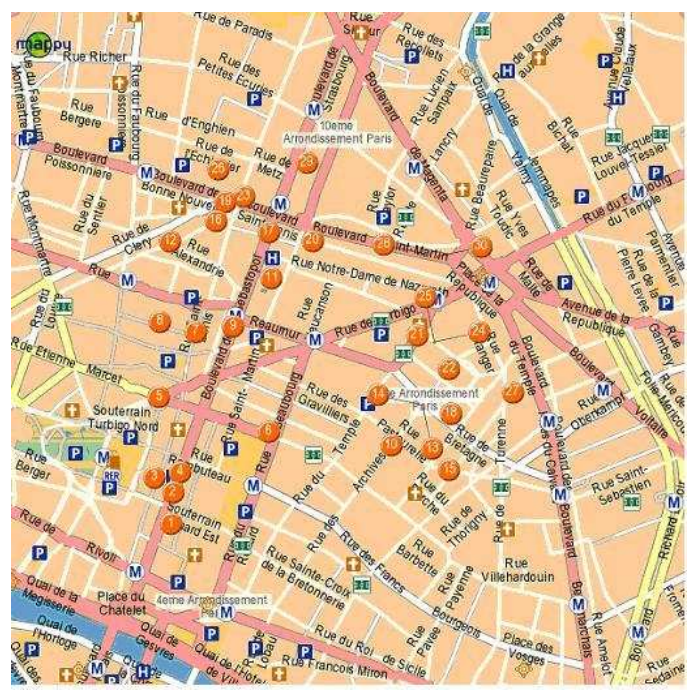

Fig. 3. 30 APs at one of Paris's Districts

The NGP-A algorithm chooses the MN's next access point according to the MN's localization and nearby signal powers. The Reply message (on Figure 5) thus consists of the next SSID, the next channel and the IP temporary address. (for the detailed information about the formats of these messages see [15]). As a result, the probe latency at the Link Layer can be reduced from $400 \mathrm{~ms}$ to $40 \mathrm{~ms}$.

\section{Extended Handover Control Function-EHCF at the Net- work Layer}

At the Network Layer, we introduce a local intelligent entity called Extended Handover Control Function $(E H C F)$ which should be capable of controlling its attached Access 


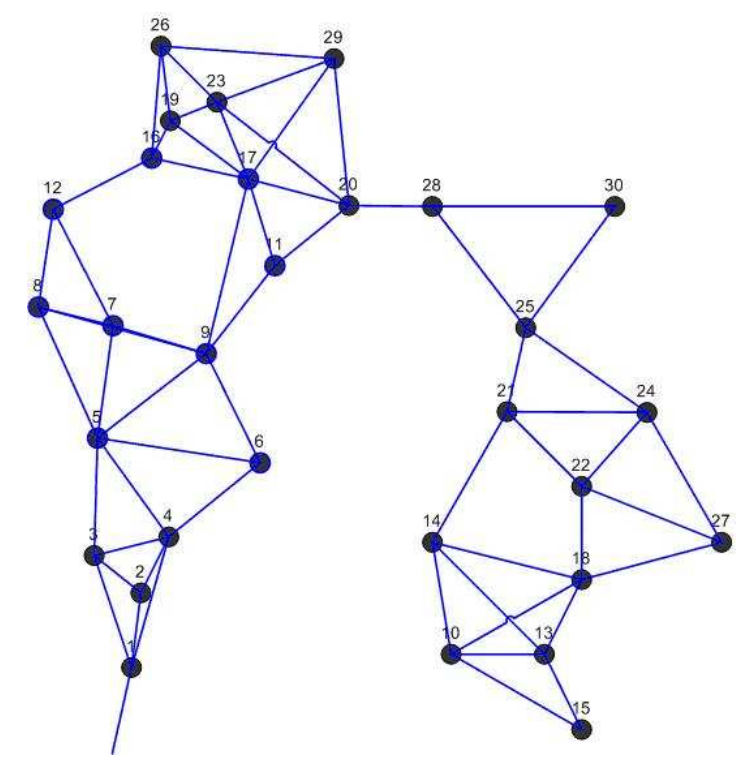

Fig. 4. 30 APs Graphical Map correspond to one of Paris's Districts

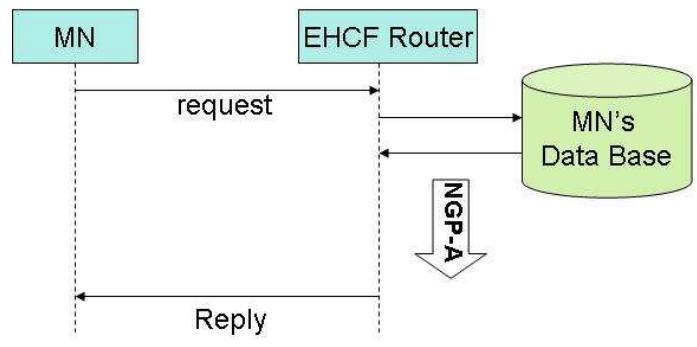

Fig. 5. Procedure of the NGP prediction

Routers (ARs), Access Points (APs) and Mobile Nodes $(M N s)$. As shown on Figure 2, linked directly with its ARs, each $E H C F$ router reserves a list of all available IP local addresses. An $E H C F$ router also generates and updates periodically a second list which records the used ARs/APs/IP addresses. By comparing these two lists, the $E H C F$ router can find a potential duplicate IP address (collision) in its domain. Then, this $E H C F$ router can withdraw this potential duplicate IP address or can ask a concerned sub-node to change its IP address. In this way, the $E H C F$ router enables to insure an unique IP address to a $M N$ without DAD.

Furthermore, an EHCF router could exchange both some local information with its ARs/APs/MNs and some external information with other $E H C F$ routers. To realize our $E H C F$ proposal, we propose six new messages: MN Request $(M N$ Req), MN Reply (MNRep), HCF Request (HCFReq), HCF Reply (HCFRep), Connection Established Information (CEInf) and Handover Finished Confirmation (HFCon) messages (for the detailed information about the formats of these messages see [15]). In order to minimize the packet loss during a handover, an EHCF router stores packets into a buffer until the $M N$ is really attached to the new IP address. The entire handover procedure is displayed on Figure 6.

1) EHCF Procedure: We first recall that HCFReq/HCFRep messages are used between $E H C F$ routers for extra-domain handovers. Each $E H C F$ router must record and update its

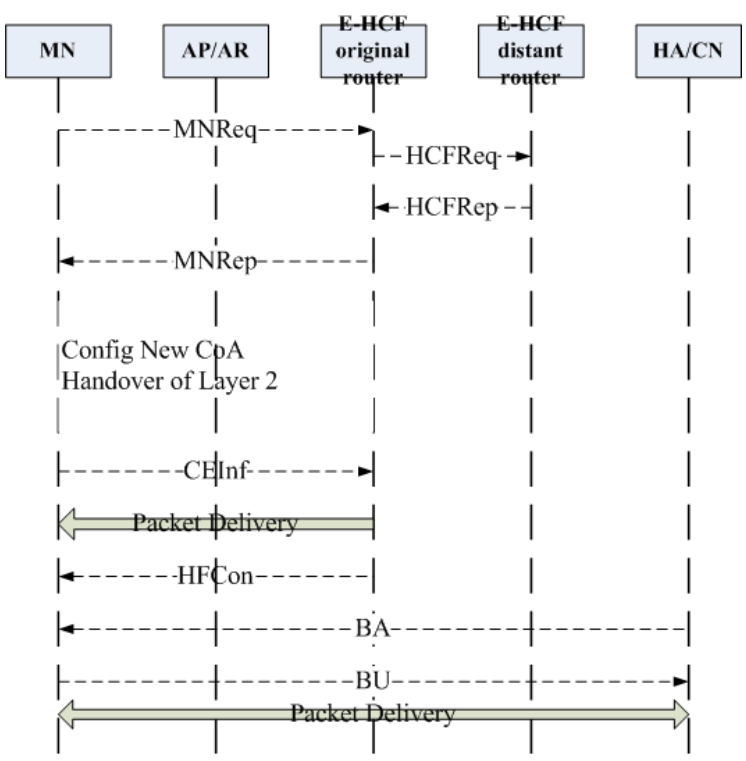

Fig. 6. EHCF Procedure (EHCF original router is an attached router with an $\mathrm{EHCF}$ function; the $\mathrm{EHCF}$ distant/remove router is a router with who an EHCF original router can communicate)

database periodically. This database helps to decide an unique new IP configuration in order to adapt for $M N$ movements without the $D A D$ phase during a handover.

As illustrated on Figure 6, the EHCF procedure is composed of the following steps:

- Moving in the network, if the threshold of the received signal strength is overstepped, the $M N$ begins to probe the neighbor $A R / A \mathrm{P}$ 's information, including the signal strength, some IP addresses, $A P$ 's $B S S I D s, A R$ interface addresses and the sub-network prefix. Then the $M N$ sends a MNReq message to its $E-H C F$ original router (via its $A R / A P$ ) to report this information.

- Receiving the MNReq message, the AR stops to forward all the packets sent to the $M N$ and forwards them to its $E-H C F$ original router in order to avoid the packet loss during the handover procedure.

- Receiving the MNReq message, the E-HCF original router decides to which $A R / A P$ the $M N$ will be associated. The choice of the $A R / A P$ is mostly based on database obtained with periodic exchange messages from an $E$ $H C F$ router to another (HCFReq and HCFRep messages) or with periodic exchange messages from ARs/APs/MNs. For example, if the number of registered $M N \mathrm{~s}$ in one $A R$ or $A P$ has reached a limit, the $E H C F$ original router will not attach the $M N$ to this saturated $A R$ or $A P$. After making the previous decision, the $E H C F$ original router sends to the MN a MNRep message which consists of a new $A P$ 's $B S S I D$, an $A R$ interface address, a sub-network prefix and a new IP address.

- With the MNRep message, the MN can obtain its new CoA and configure it automatically.

- The $M N$ sends a CEInf message to its EHCF original router to confirm its new attachment.

- After receiving the CEInf message, the EHCF original 
router transfers the buffered packets to the $M N$ 's new $C o A$. Then, the EHCF original router sends an HFCon message to end the handover procedure.

- The $M N$ can then exchange Binding Update $(B U)$ and Binding Acknowledgement $(B A)$ messages with its home agent and its correspondent node.

As shown in the $E-H C F$ procedure, a $M N$ can obtain its new $C o A$ before it really attaches to its next $A R / A P$. Moreover, any $D A D$ latency (about $1000 \mathrm{~ms}$ ) is avoided. Thus, the $E H C F$ approach allows the reduction of both the traditional handover latency and the packet loss. The handover performance is thus optimized compared to a traditional approach.

\section{NGP-EHCF PERFORMANCE ESTIMATION}

The NGP-EHCF performance estimation has been evaluated in terms of the total handover latency and of the packet loss with an analytical model. This model allows us to compare our $N G P$-EHCF handover performance with the standard handover of the MIPv6 protocol.

\section{A. NGP-EHCF Latency Analysis}

According to the handover procedure on Figure 3, we cite the following latency notations to estimate the handover latency:

- $L_{E H C F}$ : Total handover latency with the EHCF approach.

- $L_{N G P}$ : Latency due to the $M N$ 's handover at the Link Layer.

- $L_{M N R e q}$ : Latency for a $M N$ to send a MNReq message to its $E-H C F$ original router.

- $L_{d e c}$ : Latency necessary to an EHCF router to decide which $A R / A P$ the $M N$ should be attached (including the short delays to send an HCFReq message and to receive an HCFRep message).

- $L_{M N R e p}$ : Latency for an E-HCF router to send a MNRep message to the $M N$.

- $L_{C N i n f}$ : Latency necessary for a $M N$ to auto-configure its new $\mathrm{CoA}$.

- $L_{\text {conf }}$ : Latency due to the fact that an $E H C F$ router sends buffered packets and a HFCon message.

- $L_{B U / B A}$ : Binding Update/Binding Acknowledgement latency.

The average overall $E H C F$ handover latency $L_{E H C F}$ can be summed as following:

$$
\begin{aligned}
& L_{E H C F}= \\
& \quad L_{N G P}+L_{M N R e q}+L_{d e c}+L_{M N R e p}+ \\
& \quad L_{C N i n f}+L_{c o n f}+L_{B U / B A}
\end{aligned}
$$

As this $L_{E H C F}$ depends upon the mobile link bandwidth and the computation capacity of each entity in the wireless network, we summarize the parameter values used in our numerical analysis in Table I.
TABLE I

Parameter Setting

\begin{tabular}{||l|l|l||}
\hline Parameter & Value & Comment \\
\hline \hline Channel scan time & $50 \mathrm{~ms}$ & MIPv6 standard \\
\hline BU/BA latency & $140 \mathrm{~ms}$ & MIPv6 standard \\
\hline Wireless link bandwidth & $5.5 \mathrm{Mb} / \mathrm{s}$ & IEEE $802.11 \mathrm{~b}$ \\
\hline AR computation capacity & $20 \mathrm{Mb} / \mathrm{s}$ & general router \\
\hline MN computation capacity & $10 \mathrm{Mb} / \mathrm{s}$ & PC computation capacity \\
\hline MNReq message size & $72 \mathrm{byte}$ & NGP-EHCF approach \\
\hline MNRep message size & 45 byte & NGP-EHCF approach \\
\hline HCFReq message size & 45 byte & NGP-EHCF approach \\
\hline HCFReq message size & 45 byte & NGP-EHCF approach \\
\hline CEInf message size & 45 byte & NGP-EHCF approach \\
\hline HFCon message size & 24 byte & NGP-EHCF approach \\
\hline
\end{tabular}

\section{B. Numerical Results of the Total Handover Latency}

With the parameters of Table I, we give a latency comparison between the standard handover latency and the $N G P$ $E H C F$ latency according to equation (1). These latencies are functions of the wireless link bandwidth and of the computation capacity. For example, the $L_{M N R e q}$ latency can be numerically estimated as following: with a $10 \mathrm{Mb} / \mathrm{s}$ computation capacity, a $M N$ needs $57.6 \mu$ s to generate a 72-byte MNReq message, whereas, $28.8 \mu$ s are required for an Access Router. Putting this 72-byte message on a 9kb/s GSM network, requires about $64 \mathrm{~ms}$, so that the global of $L_{M N R e q}$ is about $64 \mathrm{~ms}$

On Figure 7, the standard MIPv6 handover latencies (upper curve) and the NGP-EHCF handover latencies as function of the number of handovers are displayed. With an IEEE $80.211 \mathrm{~b}$ wireless network, the average of the NGP-EHCF handover latency is about $200 \mathrm{~ms}$. This average NGP-EHCF handover latency is validated by our simulation results on OPNET illustrated on Figure 8.

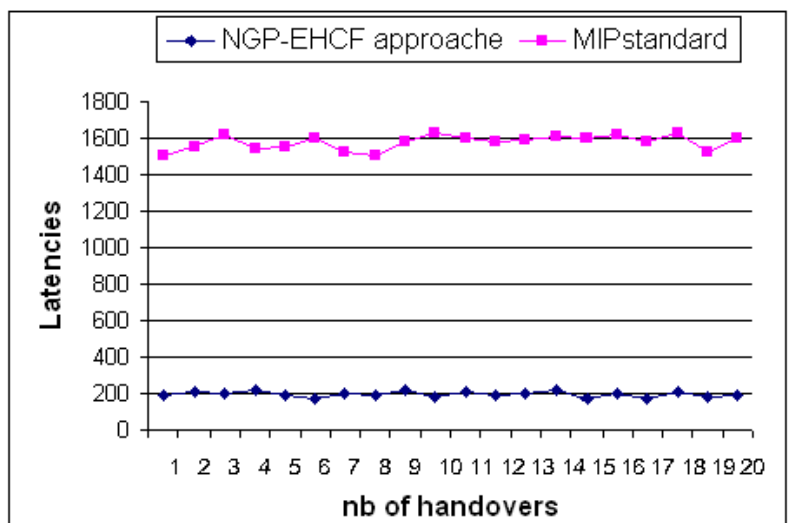

Fig. 7. NGP-EHCF handover latencies and the standare MIPv6 handover latencies with IEEE802.11b

Using the NGP-EHCF cross-layer solution, the latency reduction from $1620 \mathrm{~ms}$ to less $200 \mathrm{~ms}$ comes from avoiding the probe process at the Link Layer and the $D A D$ phase at the Network Layer.

\section{NGP-EHCF Loss}

In terms of packet loss with the NGP-EHCF approach, packets can be stored into a buffer during the handover (see 


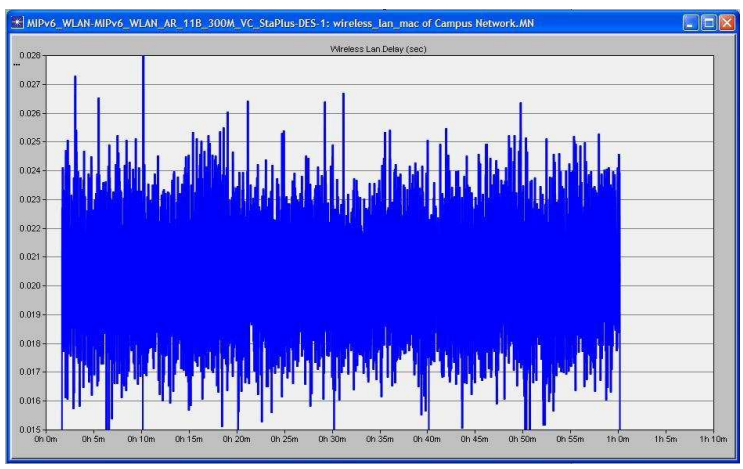

Fig. 8. NGP-EHCF handover latency by simulation

subsection II-C.1). Figure 9 illustrates the comparison of packet loss rates between the NGP-EHCF approach and the MIPv6 standard. The upper curve represents the number of lost packets with the MIPv6 standard (38 packets received out of 100 emitted packets), where the bottom curve with NGP-EHCF approach (68 packets received out of 100 emitted packets). This gives a typical $30 \%$ gain with the NGP-EHCF approach.

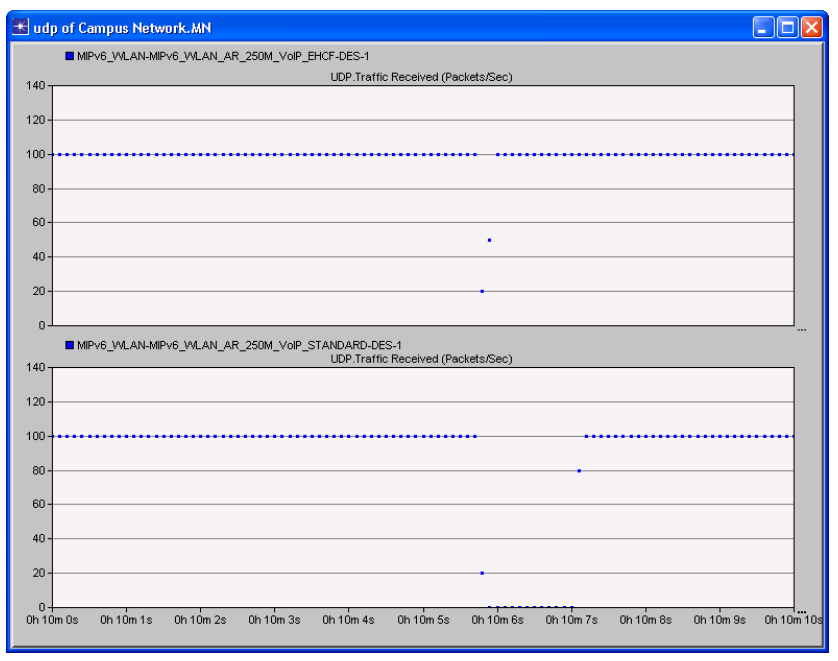

Fig. 9. Comparison of loss rates between the NGP-EHCF approach and the MIPv6 standard by simulation

\section{CONCLUSION}

In order to improve the handover performance for the Mobile IPv6, this paper proposes a cross-layer solution-NGP$E H C F$ based on a location graph at the Link Layer (NGP) and on a control function at the Network Layer (EHCF). The NGP$E H C F$ approach allows to collect and store some link and network data in order to anticipate some handover operations. Regarding the classical Mobile IPv6 handover performance, our numerical results validated by simulations show that the $N G P$-EHCF approach enables to decrease significantly both the total handover latency and the packet loss.

\section{REFERENCES}

[1] C. Perkins, "IP Mobility support for IPv4," RFC 3220, IETF, January 2002.
[2] D. Johnson, C. Perkins, and J.Arkko, "Mobility Support in IPv6," RFC 3775, June 2004

[3] G. Z. Wei, A. Wei, K. Xu and H. Deng, "Handover Control Function Based Handover for Mobile IPv6," In Proceedings of Workshop of Evolution towards Next Generation Internet (ICCS 2006), University of Reading, UK, 28-31 May 2006.

[4] C. E. Perkins, Tutorial Mobile Networking through Mobile IPv4, Sun Microsystems, http://www.computer.org/internet/v2n1/ perkins.html. 2005

[5] H. Soliman, C. Castelluccia, K. Malki, and L. Bellier, "Hierarchical Mobile IPv6 mobility management (HMIPv6)," RFC 4140, August 2005.

[6] H. Fathi, S.H. Shin, K. Kobara, and al, "Leakage-Resilient security Architecture for Mobile IPv6 in Wireless overlay Networks," IEEE Journal on Selected Areas in Communications, vol. 23, No. 11, pp.21822193, November 2005.

[7] Wei Kuang Lai and Jung Chia Chiu, 'Improving Handoff Performance in Wireless Overlay Networks by Switching between Two-Layer IPv6 and One-Layer IPv6 Addressing," IEEE Journal on Selected Areas in Communications, vol. 23, No. 11, pp. 2129-2137, November 2005.

[8] R. Koodli, Ed. ”Fast Handovers for Mobile IPv6," RFC 4068, July 2005.

[9] H. Chaouchi and P. Antunes, "Pre-handover Signaling for QoS Aware Mobility Management," International Journal of Network Management, No. 14, pp.367-374, 2004.

[10] Y. Bi, P. Iyer, ”An Integrated IP-layer Handover Solution for Next Generation IP-based Wireless Network", IEEE Vehicular Technology Conference VTC2004-Fall, Vol. 6, pp.3950-3954, Los Angeles, USA, 2004

[11] J. Kristiansson and P. Parnes, "Application-layer Mobility Support for Streaming Real-time Media," Wireless Communications and Networking Conference, Vol.1, pp.268-273, Atlanta, USA, 2004.

[12] S. Kashihara, K. Iida and al, "End-to-End Seamless Handover Using Multi-path Transmission Algorithm," International Conference on Internet Computing 2002, IC'02, pp. 1-7, Las Vegas, USA, 2002.

[13] "IPv6 Address Allocation and Assignment Policy," doc no. ripe267, http://www.ripe.net/ripe/docs/ipv6policy. html,January 2003

[14] "IEEE 802.11f: Recommended Practice for Multi-Vendor Access Point Interoperability via an Inter-Access Point Protocol Access Distribution Systems Supporting IEEE 802.11 Operation", IEEE Standard 802.11, January 2003

[15] Guozhi Wei, "Handover Optimisation using E-HCF Method for the Mobile IPv6 protocol," thesis, University of Paris XII, France, February, 2008.

[16] J. Bournelle and M. Laurent-Marknavicius, "Adaptation et implementation de Diameter/AAA pour Mobile IPv6," http://www-rp.lip6. fr/dnac/5.1-bournelle-article.pdf, 2004

[17] A. Patel, K. Leung et al, "Authentication Protocol for Mobile IPv6," RFC 4285, January, 2006.

[18] J. Arkko, V. Devarappli and F. Dupont, "Using IPSec to Protect Mobile IPv6 Signaling Between Mobile Nodes and Home Agents," RFC 3776, June 2004.

[19] A. Mishra, A. Shin and W. Arbaugy, "An Empirical Analysis of the IEEE 802.11 MAC Layer Handover Process," ACM SIGCOMM Computer Communications, Rev. vol. 33, no. 2, Apr. 2003

[20] B. Aboda, "Fast Handover Issues," IEEE-03-155r0-I, IEEE 802.11 Working Group, Mar. 2003

[21] P.J. Huang and Y.C. Tseng, "A Fast Handover Mechanism for IEEE 802.11 and IAPP Networks," IEEE 63rd Vehicular Technology Conference, Melbourne, Australia, 7-10 May, 2006

[22] S. Pack, H. Jung, T. Kwon and Y.Choi, "A Selective Neighbor Caching Scheme for Fast Handover in IEEE 802.11 Wireless Networks," International Technical Conference on Circuits/Systems, Computers and Communications, Japan, July 2004 